\title{
In situ diffraction informed by structure prediction for the discovery of novel functional materials.
}

\author{
J. B. Parise ${ }^{1}$ (John.Parise@stonybrook.edu) Department of Geosciences, Stony Brook \\ University, Stony Brook, NY, 11794-2100.
}

Discoveries of new inorganic and hybrid materials with enhanced properties drive advances in condensed matter science and engineering. The strategies employed in exploratory synthesis often relying on "cook-and-look" techniques and empirically derived structureproperty relationships. In those cases where the synthesis is onerous - recovery of samples from high pressure conditions for example - and/or where post synthesis testing is arduous - determination of sorption isotherms, for example -in situ techniques, coupled to theory, greatly narrows searches for new materials and enhanced properties. The talk will review two generic areas of research positively impacted by in situ techniques: 1) The application of modern structure prediction tools and in situ synchrotron x-ray diffraction to enhance the throughput for pressure exploratory synthesis ${ }^{1}$. 2) The application of database search, simultaneous calorimetry-XRD and in situ gas loading with synchrotron-based x-ray powder scattering to identify optimal materials for gas separation ${ }^{2}$.

The systematic search for novel semiconductors for photocatalytic overall water splitting is a representative case where quench high-pressure synthesis informed by theoretical calculations is useful, and sometimes necessary, in order to efficiently identify and synthesize target compounds. The increasing availability of community-based databases and $a b$ initio theory provides new opportunities for structure search based on desired properties, such as band gap and band edge position. By employing in situ laboratory and synchrotron-based x-ray scattering techniques, synthesis target lists can be rapidly surveyed to speed up the discovery and optimization of novel compounds.

Many of the strategies developed can be adapted to a varied of problems in materials chemistry.

\section{References:}

1. Woerner, W. R.; Qian, G. R.; Oganov, A. R.; Stephens, P. W.; Dharmagunawardhane, H. A. N.; Sinclair, A.; Parise, J. B. Combined Theoretical and in Situ Scattering Strategies for Optimized Discovery and Recovery of HighPressure Phases: A Case Study of the $\mathrm{GaN}-\mathrm{Nb}_{2} \mathrm{O}_{5}$ System Inorg. Chem. 2016, 55, 3384-3392.

2. Banerjee, D.; Simon, C. M.; Plonka, A. M.; Motkuri, R. K.; Liu, J.; Chen, X. Y.; Smit, B.; Parise, J. B.; Haranczyk, M.; Thallapally, P. K. Metal-organic framework with optimally selective xenon adsorption and separation Nat. Commun. 2016, 7, article 11831 